\title{
Bioadhesion of Polyion Complex (PIC) Hydrogels Composed of Amphiphilic Phospholipid Polymers
}

\author{
Mizuna Kimura ${ }^{\mathrm{a}}$, Madoka Takai ${ }^{\mathrm{a}, \mathrm{c}}$ and Kazuhiko Ishihara ${ }^{\mathrm{ab}, \mathrm{b}, \mathrm{c}, *}$ \\ ${ }^{a}$ Department of Materials Engineering, ${ }^{b}$ Department of Bioengineering, and ${ }^{c}$ Center for NanoBio Integration (CNBI), \\ The University of Tokyo, 7-3-1 Hongo, Bunkyo-ku, Tokyo 113-8656, Japan \\ Fax: 81-3-5841-8647, e-mail: ishihara@mpc.t.u-tokyo.ac.jp
}

\begin{abstract}
To investigate the tissue adhesive function of a hydrogel composed of biocompatible amphiphilic polymers, random and graft polymers were prepared from 2-methacryloyloxyethyl phosphorylcholine (MPC), electrolyte monomers and hydrophobic $n$-butyl methacrylate (BMA). The results of the fluorescence study suggested enhanced electrostatic interaction in the graft polymers compared to the random polymers. This is due to the strategically designed architectures and the hydrophobic BMA units. The results of the cytotoxicity test showed that the cytotoxicity of the MPC polymers was lower than that of glutaraldehyde, which is a crosslinking agent in the aldehyde-type tissue adhesives and is known to cause serious side effects. The cationic MPC polymers demonstrated higher cytotoxicity compared to the anionic ones, which demonstrated no significant cytotoxicity. The tissue adhesion of the PIC hydrogels was lower than that of a commercially available fibrin glue. However, the tissue adhesive strength increased with an increase in the polymer concentration and could be controlled by the water content of the hydrogel. In addition, balance of the electrolyte units for the PIC formation and the tissue adhesion is crucial for expression of adhesion. Although further investigation of the biocompatibility is required, it can be concluded that the PIC hydrogels formed by the amphiphilic MPC polymers can be a promising tissue adhesive which demonstrate tissue adhesion and biocompatibility according to the architectures and chemical structures.

Key words: tissue adhesive, hydrogel, polyion complex, 2-methacryloyloxyethyl phosphorylcholine polymer, biocompatibility
\end{abstract}

\section{INTRODUCTION}

An amphiphilic polymer forms a structure in aqueous medium via various intermolecular forces such as hydrophobic interaction, hydrogen bonding, electrostatic interaction, and van der Waals force. Regarding one of the amphiphilic polymers containing 2-methacryloyloxyethyl phosphorylcholine (MPC) units, several studies have been reported on the behavior of the MPC polymers in aqueous medium $[1,2]$. We also observed intermolecular complex formation from water-soluble MPC polymers via a combination of hydrophobic interaction and hydrogen bonding. For example, a mixture of aqueous solutions containing an MPC polymer with hydrophobic groups and that with carboxylic acid groups can form an intermolecular complex and finally generate a hydrogel [3]. Because the polymer chains containing carboxylic acid groups diffuse into the hydrophobic domains formed by the hydrophobic groups and the number of carboxylate anions is decreased due to the lowered polarity, the resulting hydrogen bonding formation plays an important role in this gelation mechanism. Therefore, control of hydrogel formation and dissociation could be possible according to a change in $\mathrm{pH}$ of the medium. We have investigated the MPC polymer hydrogel as a material for a $\mathrm{pH}$-responsive drug delivery system [4]. Moreover, introduction of multivalent cationic ions leads to another crosslinking formation between the carboxylate anions and the cations, and enables control of the dissociation time. Thus, the MPC polymer hydrogel is expected to be a promising soft biomaterial for various applications such as an antiadhesive material for the healing process after a physical surgery [5].

In addition, tissue adhesion is an important issue to control interaction with cells by a polymeric structure. In terms of adhesion to tissues, we hypothesized that utilization of electrostatic interaction is effective among the intermolecular forces between the surfaces of the material and the tissue. In addition, it is advantageous to apply polymers which can spontaneously form a hydrogel for this purpose, because solidification from a liquid is required in the adhesion process. Commercially available synthetic adhesive agents containing aldehyde compounds or cyanoacrylates cause chronic inflammation for reactive low-molecular-weight substances [6]. Natural fibrin glues have a potential for virus infection of biological materials [7]. Lowering these risks of polymeric adhesive materials will be necessary.

Thus, we developed novel amphiphilic polyelectrolytes with several architectures and chemical structures based on MPC polymers. The MPC polymers are well-known to have excellent biocompatibility $[8,9]$. We evaluated the physicochemical properties of the polyion complex (PIC) hydrogels with attention to the molecular structures and the aggregation states. The biological properties such as biocompatibility and adhesive strength were also investigated.

2. MATERIALS AND METHODS

2.1 Materials 
MPC was synthesized using a previously reported method and purified by recrystallization from acetonitrile [10]. 2-Methacryloyloxyethyl trimethylammonium chloride (MTC) was purchased from SIGMA-Aldrich (St. Louis, USA) as a $75 \mathrm{wt} \%$ aqueous solution and used without further purification. Sodium 2-methacryloyloxyethyl sulfonate (MSS) was purchased from Wako Pure Chemical Industries, Ltd., (Osaka, Japan) and was used as received. $n$-Butyl methacrylate (BMA) was purchased from Kanto Chemical (Tokyo, Japan) and purified under reduced pressure (bp $63^{\circ} \mathrm{C} / 24$ $\mathrm{mmHg}$ ). A monomer with a photoreactive group, vinylbenzyl $N, N$-diethyldithiocarbamate (VBD) were synthesized using a reported method and purified by recrystallization from methanol [11]. All other reagents were of extra-pure reagent grade.

2.2 Preparation of amphiphilic MPC polymers

The random MPC polymers were synthesized by a conventional radical polymerization technique. As a photoreactive prepolymer, poly(MPC-co-VBD) (PMV) was synthesized with 2,2'-azobisisobutyronitrile by a conventional radical polymerization. MPC and VBD were dissolved in a mixture of ethanol and dioxane (7/3 by volume). After argon bubbling, the polymerization was carried out at $60^{\circ} \mathrm{C}$ for a given time. Chloroform was used to precipitate the formed polymer.

For synthesis of the cationic graft polymer, $g B N$, the MTC and BMA were dissolved in methanol. After argon bubbling, the photoinduced polymerization was carried out. The formed polymer was precipitated in chloroform. For the anionic graft polymer, gBS, the MSS and BMA were dissolved in a 40 vol\% ethanol aqueous solution. The formed polymer was precipitated in ethanol.

\subsection{Fluorescence study}

The fluorescence spectrum measurement was conducted (the excitation wavelength, $350 \mathrm{~nm}$ ). A fluorescence probe, sodium 1-anilino-naphthalene-8-sulfonate (ANS) was used and the concentration was $1.0 \times 10^{-5}$ $\mathrm{mol} / \mathrm{L}$. The prepared polymer samples were kept at room temperature for a night before the measurement.

2.4 Stability of random polymer PIC hydrogels

Equal amounts $(400 \mu \mathrm{L})$ of the MPC polymer solutions (5wt\%) were mixed to obtain a PIC hydrogel and kept at room temperature for a night. The hydrogel was put into a nylon mesh bag and freeze-dried. After the hydrogel was immersed in distilled water or phosphate-buffered saline (PBS; $0.15 \mathrm{M}, \mathrm{pH} 7.1,30 \mathrm{~mL}$ ) for 15 hours, the hydrogel was freeze-dried again to evaluate the weights before and after the immersion.

2.5 Cytotoxicity test

The cytotoxicity of the MPC polymers was evaluated by culturing L929 fibroblasts. L929 cells were seeded in a 96-well multiplate at a density of $4 \times 10^{3}$ cells/well in MEM $(50 \mu \mathrm{L})$ supplemented with L-glutamine and $5 \%$ calf serum, and incubated for 1 day at $37{ }^{\circ} \mathrm{C}$ and $5 \%$ $\mathrm{CO}_{2}$. The MPC polymers were dissolved in PBS and sterilized by filtration. The polymer solutions $(50 \mu \mathrm{L})$ were then added to the cells at different concentrations. PBS without additional reagents was used as the control. After incubation for 1 day at $37{ }^{\circ} \mathrm{C}$ and $5 \% \mathrm{CO}_{2}$, the number of surviving cells was determined using a Cell Counting Kit (Dojindo Laboratories, Kumamoto, Japan). 2.6 Adhesive strength measurement to tissue in vitro

A dura incision was sewn and placed into a pressure chamber. The suture line was left either untreated or was covered with $5 \% \mathrm{rN} / \mathrm{rS}$ mixed solution, $10 \% \mathrm{rN} / \mathrm{rS}$ mixed solutionl, or Beriplast Combi-Set (ZLB Behring, Melbourne, Australia) administered with a needle. The pressure was increased until fluid leaks were observed from the suture line.

\section{RESULTS AND DISCUSSION}

3.1 Characterization of the amphiphilic MPC polymers

The random and graft polyelectrolytes based on the MPC polymers were successively prepared. The FT-IR spectra of these polymers had corresponding IR absorption attributed to the functional groups in the polymers. We prepared the random MPC polymers with different molecular weights and compositions. The number-averaged molecular weight and weight-averaged molecular weight could be controlled by the monomerinitiator ratio. The unit compositions in the random polymers could be controlled by those in feed. The ratio of the BMA units to the electrolyte units was about $10 / 90$ in the graft polymers, which was close to the ratio in the feed. The characterization of the MPC polymers mainly used in the following study is summarized in Table I.

Excellent biocompatibility and high tissue adhesion arerequired for medical adhesives. We have designed amphiphilic graft MPC polymers with attention to two points. One is the strategic architectures to gain biocompatibility and the interaction for tissue adhesion and solidification. The local concentrations of the electrolyte monomer units increase in a graft polymer compared to a random polymer. The graft polymer has

Table I Synthetic results of the amphiphilic polymers

\begin{tabular}{|c|c|c|c|c|c|}
\hline \multirow{2}{*}{ Abb. } & \multicolumn{3}{|c|}{ Unit mole fraction in copolymer** } & \multirow{2}{*}{$\begin{array}{l}M w^{* * *} \\
\left(\times 10^{5}\right) \\
\end{array}$} & \multirow{2}{*}{$\mathrm{Mw} / \mathrm{Mn}$} \\
\hline & MPC W & $\mathrm{X}^{* * * *}$ & 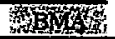 & & \\
\hline PMV & 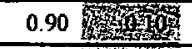 & & 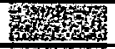 & 0.6 & 2.2 \\
\hline $\mathrm{rN}$ & 0.26 Wh & 0.74 & 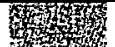 & 2.2 & 5.5 \\
\hline gBN & 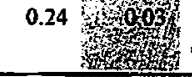 & $\begin{array}{r}0.68 \\
+25.06 \\
\end{array}$ & 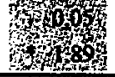 & - & - \\
\hline rS & 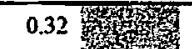 & 0.68 & 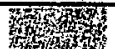 & 2.9 & 2.6 \\
\hline gBS & 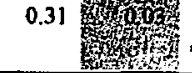 & $\begin{array}{r}0.59 \\
+16.91\end{array}$ & 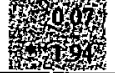 & - & - \\
\hline
\end{tabular}

- Average number of $X$ units per VBD unit. * Defermined by $\mathrm{H}-\mathrm{NMR}$. *** PEG standard, PBS

**** X $=$ MTC (cationic polymer), or MSS (anionic polymer)

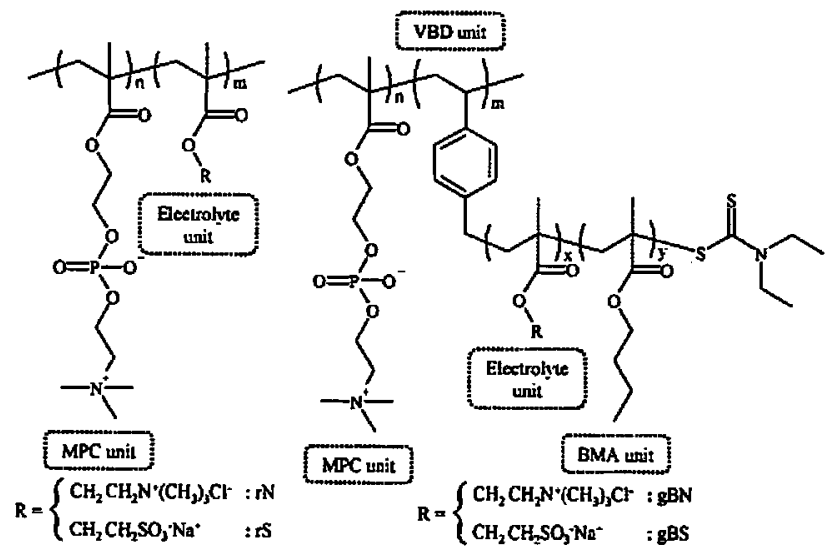

Fig. 1 Chemical structures of the MPC polymers. 
highly mobile side chains. Thus, enhancement in the interaction between the polymer and the tissue surface is expected. The local concentration of the MPC units may be accompanied by higher biocompatibility than the random polymer. The other is utilization of hydrophobic BMA units. We introduced the BMA units by random polymerization with the electrolyte monomers, because the hydrophobic unit is expected to decrease the polarity and enhance the electrostatic force. This can cause an increase in the physical crosslinking of the polymer chains of the PIC hydrogel as well as an increase in the tissue adhesive strength.

It is well-known that the aggregation of an amphiphilic polymer in aqueous medium depends on the architecture of the polymer as well as the chemical structure [12]. To evaluate the polarity of the hydrophobic domains formed by the polymer aggregates, the fluorescence spectra of ANS in the amphiphilic MPC polymers were analyzed. When an ANS molecule exists under hydrophobic condition, an increase in the fluorescence intensity and a blue shift of the luminescence maximum are observed [13]. A PIC hydrogel can be prepared from $5 \mathrm{wt} \%$ aqueous solutions of the cationic and anionic polymers, but an aggregate or a microgel can be formed at lower concentrations. We performed a fluorescence study with ANS to characterize the amphiphilic MPC polymers and the PIC hydrogels at lower concentrations. The effects of the architectures and chemical structures were observed in the graft polymers.

As shown in Fig. 2, a blue shift of the fluorescence maximum wavelength was observed in all the polymers and PIC hydrogels. Regardless of the architectures, the fluorescence maximum wavelength of the anionic polymers was higher than that of the corresponding cationic polymers. The hydrophobic domain formation occurred at a lower concentration in the graft polymer. This result indicates the effect of the strategically designed molecular structures. In other words, ion pairs could be formed and ionization could be suppressed in the graft polymers at lower concentration compared to the random polymer, due to the locally concentrated electrolyte units and the hydrophobic BMA units in the graft chains.

At high concentrations, the random and graft polymers formed bulk PIC hydrogels, and the fluorescence study could not be performed. However, it is noteworthy

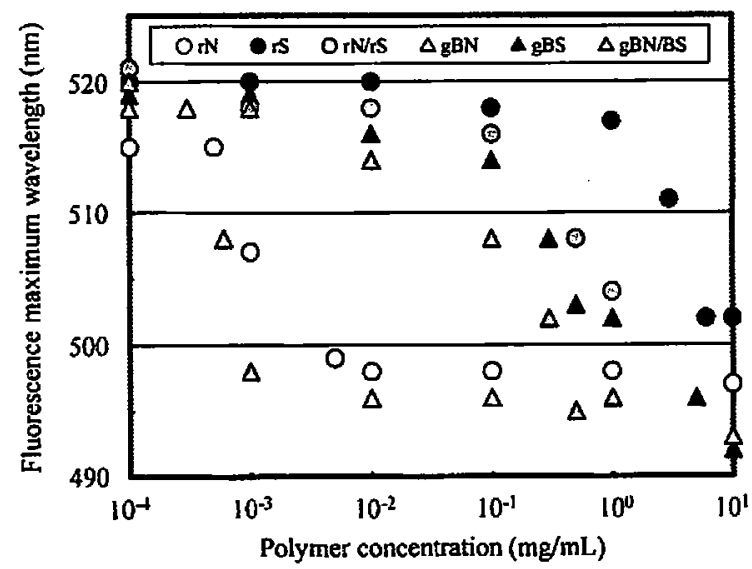

Fig. 2 The fluorescence maximum wavelength of ANS in the aqueous solutions of the polymers and the mixtures composed of the cationic and anionic polymers. that the graft PIC hydrogels showed a lower fluorescence maximum wavelength than the random PIC hydrogel. This can be explained by contribution of the hydrophobic BMA units to effective reduction in the polarity around the electrolyte units and enhancement of electrostatic interaction.

3.2 Stability of the PIC hydrogels in aqueous medium

Just after equal amounts of $5 \mathrm{wt} \%$ aqueous solutions of the $\mathrm{rN}$ and $\mathrm{rS}$ were mixed together, the mixture became clouded. After a few minutes, the PIC hydrogel was precipitated from the aqueous phase. Stability of the PIC hydrogel was investigated by immersion in a large amount of an aqueous medium. In this study, we used the random MPC polymers with different molecular weights and compositions. We also of prepared the PIC hydrogels by mixing the cationic and anionic polymers at $2 / 1,1 / 1$ and $1 / 2$, and examined their stability.

The results indicated that the stability of the PIC hydrogels was not determined mainly by the molecular weight of the MPC polymers. The PIC hydrogel showed relatively high stability when the MPC unit composition was lower than 0.5 . In addition, it is noteworthy that the mixing ratio and the mixing polymer significantly affected the stability of the PIC hydrogels. Thus, the PIC hydrogels showed low stability in an aqueous medium, when (1) the MPC unit composition was high, (2) the mixing ratio of the cationic polymer solution to the anionic polymer solution was not $1 / 1$, and (3) the MPC unit compositions of the mixed cationic and anionic polymers did not correspond to each other. When electrical charge of mixed cationic and anionic random polymers was approximately balanced, the PIC hydrogel was formed spontaneously within a few minutes and existed stably in a large amount of the aqueous medium. 3.3 Cytotoxicity of the amphiphilic MPC polymers

These PIC hydrogels can exist stably in a large amount of an aqueous medium and are expected to demonstrate a prolonged dissociation time in vivo. However, because the PIC hydrogels are crosslinked via electrostatic interaction, they can dissolve into the constitutive cationic and anionic polymers. Thus, we performed a cytotoxicity evaluation to investigate the biocompatibility of the constitutive MPC polymers in consideration of the dissociation.

The results are shown as lethal concentration 50 (LC50) in Table II. Glutaraldehyde is often used as a crosslinking agent in the aldehyde-type adhesive and is known to cause serious side effects [12]. Formaldehyde is a degradation product of the cyanoacrylate-type tissue adhesive and its cytotoxicity is reported [13]. Although cytotoxicity of formaldehyde could not be evaluated in this experiment due to its volatile, cytotoxicity of glutaraldehyde might provide an indication of that of formaldehyde. The amphiphilic MPC polymers showed lower cytotoxicity than glutaraldehyde.

Table II Cytotoxicity of the MPC polymers

\begin{tabular}{lc}
\hline & LC50 $(\mathrm{mg} / \mathrm{mL})$ \\
\hline Glutaraldehyde & 0.0008 \\
rN & 0.071 \\
gBN & 0.014 \\
rS & $>10$ \\
gBS & $>10$ \\
\hline
\end{tabular}


Compared to the cationic polymers, the biocompatibility of the anionic polymers was high regardless of the difference in the architectures. Although the cytotoxicity of a polymer depends on various factors such as molecular weight, charge density and chemical structure, anionic polymers generally demonstrate higher biocompatibility than cationic polymers [14], and these results also agreed with this general tendency.

On the other hand, the cytotoxicity of the cationic polymers depended on the architectures. The graft polymer, gBN, demonstrated higher cytotoxicity than the random polymer, $\mathrm{rN}$, although (1) the hydrophobic BMA units were expected to demonstrate lower cytotoxicity by suppressing dissociation of the MTC units, and (2) the biocompatible MPC units in the main chains were expected to contribute to lower cytotoxicity. A possible reason for this is higher mobility of the graft chains, that is, the graft chains may be more likely to interact with the cell surface and cause cytotoxicity. Several reports of cationic MPC polymers have been published. Palmer et al. reported that the incorporation of charge does not significantly increase the propensity of the polymer to bind and activate platelets from the blood cause any inflammatory response [15]. However, Rose et al. suggested that it is possible that an increase in electrostatic interaction, due to increased charge, could result in cell death because the cytotoxicity of the cationic MPC polymers could also influence cell adhesion [16]. They also argued that the free polymer itself might be cytotoxic.

The cytotoxicity of the PIC hydrogels may be distinct from that of the respective polymers, because the dissociation of the polymers can be suppressed and that the cytotoxicity of the cationic polymers, for example, can be lowered by the presence of anionic polymers. Thus, further investigation is necessary to predict the biocompatibility of the PIC hydrogel in vivo.

3.4 Adhesion strength measurement to tissue in vitro

When the swollen $\mathrm{rN} / \mathrm{rS} \mathrm{PIC}$ hydrogel was pushed between the fingers, the surface was highly lubricating at first. After pushing for about a minute, the hydrogel started to adhere to the finger skin. Because the water content of decreased, the hydrogel adsorb water from the surrounding tissues and started to interact with them. This can result in expression of bioadhesion. However, we could not investigate the adhesion of the graft polymer PIC hydrogel, because it was so rigid that they demonstrated no adhesion between the finger skins. Although the high mobility of the graft chains effectively functioned to form a PIC hydrogel, there are not enough electrolyte units to demonstrate adhesion. Balance of the electrolyte units for the PIC formation and the tissue adhesion can determine adhesion.

Thus, the adhesive strength of only the PIC hydrogel composed of random polymers was measured. The results are shown in Table III. Compared to the $5 \mathrm{wt} \%$

Table III Adhesive strength of the PIC hydrogels

\begin{tabular}{lc}
\hline & Adhesion strength (kPa) \\
\hline No adhesive & $1.3 \pm 0.6$ \\
$\mathrm{rN} / \mathrm{rS} 5 \mathrm{wt} \%$ & $6.3 \pm 4.0$ \\
$\mathrm{rN} / \mathrm{rS} 10 \mathrm{wt} \%$ & $14.7 \pm 3.1$ \\
Beriplast & $25.0 \pm 3.0$ \\
\hline
\end{tabular}

rN/rS PIC hydrogel, the 10 wt\% PIC hydrogel demonstrated a higher adhesive strength, although the polymer solution was so viscous that a 20 wt\% solution could not be applied with a needle. That is, an increase in the polymer concentration resulted in an increase in the adhesive strength of the PIC hydrogel. However, the adhesive strength of the $10 \mathrm{wt} \% \mathrm{rN} / \mathrm{rS}$ PIC hydrogel was lower by about $40 \%$ than that of a fibrin glue, Beriplast $\Theta$. It is widely accepted that the tissue adhesive force of the fibrin glue is not sufficient for utilization in vivo. Thus, enhancement of the adhesive force of the PIC hydrogel is necessary.

\section{CONCLUSIONS}

The properties of the PIC hydrogels depended on the strategically introduced factors such as the hydrophobic BMA units, the locally concentrated electrolyte units and biocompatible MPC units. In particular, the MPC polymers demonstrated lower cytotoxicity than glutaraldehyde. Although the PIC hydrogels composed of the random polymers demonstrated adhesion, control of the water content and the balance of the electrolyte units for the PIC formation and the tissue adhesion is crucial for expression of adhesion.

\section{REFERENCES}

[1] K. Ishihara, Y. Iwasaki and N. Nakabayashi, Polym. $J ., 31,1231-6$ (1999).

[2] T. Kitagawa, R. Iwase, K. Ishihara, T. Yamaoka and A. Murakami, Chem. Lett., 11, 1478-79 (2005).

[3] M. Kimura, K. Fukumoto, J. Watanabe and K. Ishihara, J. Bionzater. Sci. Polymer Edn, 15, 631-44 (2004).

[4] K. Nam, J. Watanabe and K. Ishihara, Int. J. Pharma., 275, 259-69 (2004).

[5] M. Kimura, T. Konno, M. Takai, N. Ishiyama, T. Moro and K. Ishihara, Key Eng. Mater., 342-3, 777-80 (2007).

[6] Y. C. Tseng, Y. Tabata, S. H. Hyon and Y. Ikada, $J$. Biomed. Mater. Res., 24, 1355-67 (1990).

[ 7] H. Iwata, S. Matsuda, K. Mitsuhashi, E. Itoha and Y. Ikada, Biomaterials, 19, 1869-76 (1998).

[8] M. Kimura, M. Takai and K. Ishihara, J. Biomed. Mater. Res., 80A, 45-54 (2007).

[9] S. Sawada, S. Sakaki, Y. Iwasaki, N. Nakabayashi and K. Ishihara, J. Biomed. Mater. Res., 79A, 476-84 (2006).

[10]K. Ishihara, T. Ueda and N. Nakabayashi, Polym. J., 22, 355-60 (1990).

[11]Y. Nakayama and T. Matsuda, Macromolecules, 29, 8622-30 (1996).

[12] H. Mori, and A. H. E. Müller, Progr. Polym. Sci., 28, 1403-39 (2003).

[13] V. E. Syakhovich, D. A. Parul, E. Y. Ruta, B. A. Bushuk, and S. B. Bokut, Biochem. Biophys. Res. Commun., 317, 761-7 (2004).

[14] K. Nishida, K. Mihara, T. Takino, S. Nakane, Y. Takakura, M. Hashida and H. Sezaki, Pharm. Res., 8, 437-44 (1991).

[15] R. R. Palmer, A. L. Lewis, L. C. Kirkwood, S. F. Rose, A. W. Lloyd, T. A. Vick and P. W. Stratford, Biomaterials, 25, 4785-96 (2004).

[16] S. F. Rose, A. L. Lewis, G. W. Hanlon and A. W. Lloyd, Biomaterials, 25, 5125-35 (2004). 